\title{
CMBF-based dynamic selection for heterogeneous massive MIMO systems
}

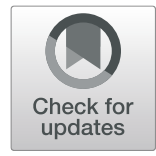

Yinghui Zhang ${ }^{1}$, Biao Zhang ${ }^{1}$, Yifan Mu' ${ }^{1}$ Kun Song ${ }^{1}$, Jing Gao ${ }^{2}$ and Yang Liu ${ }^{{ }^{*}}$

\begin{abstract}
In this paper, an optimal coordinated multipoint beamforming (CMBF) scheme is proposed for heterogeneous networks (HetNets) with massive multiple input multiple output (MIMO) systems for energy efficiency (EE), taking into account the maximum transmission power of the base station (BS) with the limitation on the power consumption of the static circuit. This paper analyzes the influence of the different parameters on the EE and derives a closed-form expression. We investigate the different cooperation schemes, considering the influence of the number of antennas of small base station (SBS) and macro base station (MBS), the quality of service (QoS), and the number of different service users. Therefore, the best parameters can be selected for optimizing EE with the different scenarios in HetNets with massive MIMO systems. Theoretical derivation and simulations show that the amount of BS antennas, achievable rate constraints, quantity of service users, and cooperative schemes all have the vital influences on the EE for the CMBF design. Therefore, the proposed optimization framework enables us to identify key system parameters, and obtain the better system design for the massive MIMO HetNets.
\end{abstract}

Keywords: Energy efficiency, Massive MIMO, HetNets, Small cell networks

\section{Introduction}

With the dramatic increase in the amount of user data in wireless communication systems, mobile users are increasing their demand for multimedia services such as superdefinition video, and wireless network traffic is showing an explosive growth trend [1]. Facing the demanding requirements of high-speed, low-latency, and high-efficiency energy systems, traditional macro-cellular networks cannot fully meet the development needs of future communications. Therefore, the next-generation network (5G) imposes higher requirements on the system capacity and the transmission rate. There has been research and standardization performance using plenty of small cells (SCs) with little radius to realize same-frequency networking [2, 3]. Therefore, heterogeneous networks (HetNets) and massive MIMO cellular systems are considered to be effective means of increasing the wireless network capacity, data rates, and energy efficiency (EE) required for 5G system [4-6]. Meanwhile,

\footnotetext{
* Correspondence: liuyangimu@163.com

${ }^{1}$ College of Electronic Information Engineering, Inner Mongolia University, Hohhot, Inner Mongolia, China

Full list of author information is available at the end of the article
}

beamforming technology $[7,8]$ and Coordinated Multipoint (CoMP) (also called multi-cell cooperation) transmission technology $[9,10]$ are effective methods for reducing interference and improving EE and have become the research focus of long-term evolution advanced (LTE-A) and 5G.

Energy consumption for information and communication technology (ICT) has been growing rapidly in recent years, making up nearly $2 \%$ of $\mathrm{CO} 2$ emissions and representing a dominant factor in global energy consumption [11]. Since the exponential increase in mobile multimedia data traffic, EE has turned into the major focus of mobile communications $[12,13]$. Therefore, green and efficient mobile communication technology has attracted the attention of the academic community and has become a key technology for future systems [14-16]. In the field of the energy consumption distribution of conventional communication systems, the network component occupies nearly $90 \%$, while the terminal component occupies about $10 \%$ of the total energy consumption [17]. Thus, achieving green communication systems will significantly reduce network energy consumption in the future. 
This paper combines beamforming and CoMP technology [18], which is called coordinated multipoint beamforming (CMBF), and some researches have defined it as multi-cell cooperative beamforming. The wireless resources are improving while suppressing the interference and the system EE is optimized through the combination of beamforming and CoMP technology. Research has been carried out in this academic field and many practical proposals have been proposed. Moreover, the CoMP between the macro base station (MBS) and the small base station (SBS) plays an important role in improving EE.

Recently, several methods of improving EE have been proposed and have had a good impact on HetNets, considering the cell radius, wireless backhaul, quantity of antennas, and user density [19-21]. However, most studies that have concentrated on the analysis of dynamic transmission power affect the EE, and static circuit power transceiver hardware component is typically ignored [22-24]. Massive MIMO and small cell networks equipped more hardware, the static power cannot be neglected $[25,26]$. Therefore, the total power in HetNets with massive MIMO is modeled with a dynamic component and a static component. In a multi-cell scenario, both inter-cell and intra-cell interference degrade the performance of a massive MIMO system [27]; with the potential development of massive MIMO and dense infrastructures employing many SBSs with corresponding hardware power consumption, CMBF is of critical importance for the EE design of HetNets [28, 29]. In [28], a dual-layer coordinated beamforming scheme was proposed by dividing the beamformer into a cell-layer one and a user-layer one. The proposed scheme performs better than zero-forcing and coordinative multiple point schemes. A beamforming design of optimizing the network EE under QoS and transmit power constraints was proposed in [29], tailoring for weak, strong, and medium cross-tier interference for HetNets. Though existing coordinated beamforming algorithms have been devoted to employing $\mathrm{EE}$, none of them considers the different achievable rate requirements, quantity of service users, and the cooperative schemes. Note that it is very important to consider various parameters for optimizing the $\mathrm{EE}$ for HetNets.

In contrast to existing studies on EE of HetNets [30, 31 , this paper not only considers the impact of the dynamic transmission power, but also the BS hardware static power dissipation. Particularly in next generation communication system, the circuit power dissipation of BS cannot be ignored. Since multiple users are served by the BS configures hundreds or even several hundreds of antennas in the meantime in a massive MIMO system, the circuit power consumption of BS will have a significant impact on the EE. In this paper, we aimed at designing MBS and SBS beamforming to improve the network EE under different MBS and SBS antennas, quality of service (QoS) requirements, and the number of users.

CMBF plays a vital part in improving EE, especially in HetNets with massive MIMO, which has been attached consensus attention in recent years. The traditional CMBF often ignores the static circuit power consumption, while some studies under the condition of fixed system parameters such as antenna number, achievable rate. However, in the actual deployment, especially for massive MIMO, the static circuit power consumption cannot be ignored, and the system parameter setting needs to be considered comprehensively. This paper comprehensively considers the influence of the achievable rate constraints, antenna, and user quantity on system EE. This paper also studies the optimal number of EE cooperative BSs under different parameters, and selects the appropriate cooperative scheme and parameters according to different system conditions and service requirements to obtain the optimal system design scheme.

Firstly, we propose the HetNets system model and formulate the optimization EE problems over the downlink CoMP. Secondly, the EE optimization algorithms considering the different cooperative SBSs, the antenna numbers of the SBS and MBS, achievable rate requirements, and the number of users are proposed. Finally, we provide the simulation results and give the conclusions.

\section{System description and model}

In this section, our system modeling assumptions are introduced, then discussing the assumptions suitability and relevance.

\subsection{Network model}

The system model is illustrated in Fig. 1. MBS and SBS with $N_{\mathrm{BS}}$ and $N_{S}$ antennas in the downlink. Only one antenna is equipped on the user.

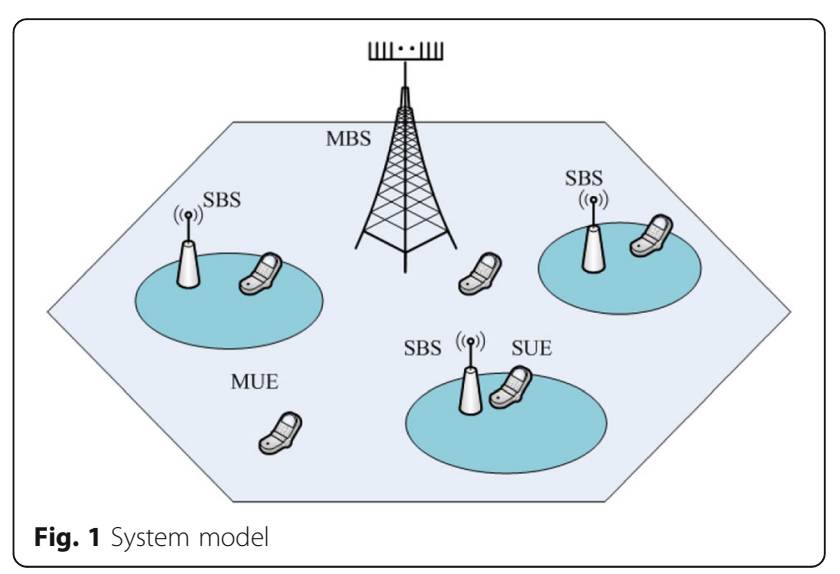


In an independent cell, the MBS and SBSs cooperate with each other to jointly serve a multi-user in HetNets. The MBS configures the massive MIMO and antenna quantity can reach into the hundreds, while a mass of antennas configured in the SBS is relatively small, generally $1 \leq N_{S} \leq 4$. Consider the TDD downlink system, the total antenna quantity is $N=N_{B S}+C N_{S}(C \leq S)$, where $C$ is the cooperative SBSs and $S$ is the total quantity of SBSs. $H$ is represented as downlink channel transmission matrix. The channel model is a block fading model, and the stationary spatial jointly Gaussian process is performed between the frames.

$$
H=\left[h_{1} h_{2} \ldots h_{k}\right]^{H} \in \mathbb{C}^{K \times N},
$$

each element of $h_{k}$ is independent identically distributed (i.i.d). The channel of the kth user can be represented as

$$
h_{k}=\left[h_{0, k} h_{1, k} \ldots h_{j, k}\right] \in \mathbb{C}^{1 \times N},
$$

the channel from the $j$ th base station to the $k$ th user is represented as $h_{j, k}$, which is complex Gaussian distribution ( mean of zero and variance of 1 ). $h_{0, k} \in \mathbb{C}^{1 \times N_{B S}}, h_{j, k} \in$ $\mathbb{C}^{1 \times N_{s}}, j=1, \ldots, S$.

\subsection{Signal model}

The $k$ th user received signals from the MBS and the $j$ th SBS are denoted as $s_{0, k}, s_{j, k}, j=1, \ldots, S$, respectively, which satisfy the zero mean and the unit variance.

$$
\mathbb{E}\left\{\left|s_{j, k}\right|^{2}\right\}=1, \forall k
$$

The beamforming vectors of the user serviced by the MBS and the SBS are defined as $w_{0, k} \in C^{N_{B S} \times 1}$ and $w_{j, k} \in$ $\mathbb{C}^{N_{S} \times 1}$, respectively. The signal sent by the BS

$$
\mathrm{s}_{j}=\sum_{k=1}^{K} w_{j, k} s_{j, k}, \quad j=0,1, \ldots, C .
$$

The $k$ th user received signal can be written as

$$
r_{k}=h_{0, k} \mathrm{~s}_{0}+\sum_{j=1}^{C} h_{j, k} \mathrm{~s}_{j}+n_{k},
$$

where $n_{k}$ is the Gaussian white noise, $n_{k} \sim C N\left(0, \sigma_{k}^{2}\right)$.

\section{Problem formulation for HetNets \\ 3.1 Energy efficiency (EE)}

The MBS and SBS are connected to each other through a backhaul link and the CMBF between the base stations. Each user can be served by more than one BS, which means that the user will receive multi-stream signals sent simultaneously by the MBS and SBS. The signal-to-interference-plus-noise ratio (SINR) can be calculated as

$$
\operatorname{SINR}_{k}=\frac{\left|h_{0, k} w_{0, k}\right|^{2}+\sum_{j=1}^{C}\left|h_{j, k} w_{j, k}\right|^{2}}{\sum_{\substack{i=1 \\ i \neq k}}^{K}\left(\left|h_{0, k} w_{0, i}\right|^{2}+\sum_{j=1}^{C}\left|h_{j, k} w_{j, i}\right|^{2}\right)+\sigma_{k}^{2}}
$$

where $\left|h_{0, k} w_{0, k}\right|^{2}$ signifies the signal power transmitted by the MBS and $\sum_{j=1}^{C}\left|h_{j, k} w_{j, k}\right|^{2}$ signifies the signal power transmitted by the SBS to the $k$ th user. In this way, thekth user's information rate is defined as

$$
R_{k}=B \log _{2}\left(1+\operatorname{SINR}_{k}\right),
$$

where $B$ is the bandwidth.

The total power consumption of the system is denoted as $P_{\text {total }}$, which is the sum of the transmission power and the circuit power $[16,32]$.

$$
P_{\text {total }}=P_{t}+P_{c},
$$

where $P_{t}$ is transmission power and $P_{c}$ is circuit power. $P_{t}$ is denoted as

$$
P_{t}=\underbrace{\frac{1}{\eta_{0}} \sum_{k=1}^{K}\left\|w_{0, k}\right\|^{2}}_{\text {Macro }}+\underbrace{\sum_{j=1}^{C} \frac{1}{\eta_{j}} \sum_{k=1}^{K}\left\|w_{j, k}\right\|^{2}}_{\text {Small Cell }}
$$

where $\eta_{0}$ and $\eta_{j}$ denote the power amplifier efficiency of the MBS and the $j$ th SBS, respectively. We assume that the efficiencies of the power amplifiers (PA) of the SBS are equal. $\eta_{j}=\eta_{\mathrm{s}}, \forall j, \frac{1}{\eta_{0}} \sum_{k=1}^{K}\left\|w_{0, k}\right\|^{2}$, and $\sum_{j=1}^{C} \frac{1}{\eta_{j}} \sum_{k=1}^{K}\left\|w_{j, k}\right\|^{2}$ denote the transmission power consumption of the MBS and the SBS, respectively.

$P_{c}$ is proportional to quantity of antennas $[21,33]$ and is signified as

$$
P_{c}=\underbrace{\rho_{0} N_{B S}}_{\text {Macro }}+\underbrace{\sum_{j=1}^{C} \rho_{j}}_{\text {Small Cell }} N_{s}+P_{0}
$$

where $\rho_{0}$ and $\rho_{j}$ represent the circuit power consumptions of the MBS and the SBS, respectively, including the cooling loss, the feeder loss, the filter loss, and the mixer loss. $P_{0}$ is the power of all the static circuits of MBS and SBSs, including power consumption values for digital to analog converter, the low noise amplifier, and analog to digital converter. The circuit power consumption is the inherent consumption of the base station [32]. Then the power consumption of base station model is denoted as 


$$
\begin{aligned}
P_{\text {total }}= & \frac{1}{\eta_{0}} \sum_{k=1}^{K}\left\|w_{0, k}\right\|^{2}+\sum_{j=1}^{C} \frac{1}{\eta_{j}} \sum_{k=1}^{K}\left\|w_{j, k}\right\|^{2} \\
& +\rho_{0} N_{\mathrm{BS}}+\sum_{j=1}^{C} \rho_{j} N_{S} .
\end{aligned}
$$

The ratio of the throughput per unit time (bit/s) to total power consumption (W) can be regarded as the EE, which can be written as

$$
E E=\frac{\sum_{k=1}^{K} E\left\{R_{k}\right\}}{P_{\text {total }}},
$$

where $E\left\{R_{k}\right\}$ is the statistical average of the achievable rate.

In this paper, we focus on improving the EE of HetNets and ensuring the achievable rate for the user.

$$
\operatorname{SINR}_{k} \geq \gamma_{k} \quad \forall k
$$

where $\gamma_{k}$ denotes the target SINR, which can be expressed by the target $R$ as

$$
\gamma_{k}=2^{R}-1 .
$$

The transmission power of each antenna for the MBS and the SBS meets the power constraint conditions as shown

$$
\sum_{k=1}^{K} w_{j, k}^{H} Q_{j, l} w_{j, k} \leq p_{l}, \quad l=1, \ldots, L_{j}
$$

where $Q_{j, l}$ is the weight matrix, which is positive semidefinite. $Q_{0, l} \in \mathbb{C}^{N_{\mathrm{BS}} \times N_{\mathrm{BS}}}, Q_{j, l} \in \mathbb{C}^{N_{S} \times N_{S}}, j=1, \ldots, S, L_{0}=N_{\mathrm{BS}}$, $L_{j}=N_{S}$, and $w_{j, k}^{H} Q_{j, l} w_{j, k}$ indicate the transmit power of the $l$ th antenna to the $k$ th user.

The EE optimization of HetNets can be equated with reducing the total power consumption when satisfying the power and the SINR constraints.

$$
\begin{array}{ll}
\min & P_{t}+P_{c} \\
\text { s.t } & \left\{\begin{array}{l}
\operatorname{SINR} R_{k} \geq \gamma_{k} \quad \forall k, \\
K \\
\sum_{k=1}^{K} w_{j, k}^{H} Q_{j, l} w_{j, l} \leq p_{j} \quad \forall j .
\end{array}\right.
\end{array}
$$

The transmission power of each antenna of the BS does not exceed the maximum transmission power, and the minimum information rate of each user is $B \log _{2}(1+$ $\gamma_{k}$ ). Thus, the EE is maximized.

\subsection{Semi-definite programming (SDP)}

The problem shown in achievable rate constraints is difficult to solve and non-convex. In this paper, semi- definite programming (SDP) is used to transform the problem into a quadratic constrained quadratic program (QCQP) problem [34].

In order to obtain presentation convenience, we introduce a new matrix.

$$
\begin{aligned}
& \mathbf{W}_{j, k}=w_{j, k} w_{j, k}^{H}, \\
& \mathbf{H}_{j, k}=h_{j, k}^{H} h_{j, k} .
\end{aligned}
$$

We can derive

$$
\begin{aligned}
& \operatorname{Tr}\left(\mathbf{W}_{j, k}\right)=\left\|w_{j, k}\right\|^{2}, \\
& \mathbf{H}_{j, k}=h_{j, k}^{H} h_{j, k} .
\end{aligned}
$$

So $\mathbf{W}_{j, k}$ is a positive semi-definite matrix, that is, $\mathbf{W}_{j, k}$ $\succeq 0$, and $\operatorname{rand}\left(\mathbf{W}_{j, k}\right) \leq 1$. The problem (16) can be rewritten as

$$
\begin{aligned}
& \min \sum_{j=0}^{S} \frac{1}{\eta_{j}} \sum_{k=1}^{K} \operatorname{Tr}\left(\mathbf{W}_{j, k}\right)+P_{c} \\
& s . t \quad\left\{\begin{array}{l}
\sum_{k=1}^{K} \operatorname{Tr}\left(\mathbf{Q}_{j, k} \mathbf{W}_{j, k}\right) \leq p_{j} \quad \forall j, \\
\frac{\sum_{j=0}^{C} \operatorname{Tr}\left(\mathbf{H}_{j, k} \mathbf{W}_{j, k}\right)}{\left.\sum_{i=1}^{K} \sum_{j=0}^{C} \operatorname{Tr}\left(\mathbf{H}_{j, k} \mathbf{W}_{j, i}\right)\right)+\sigma_{k}^{2}} \\
\begin{array}{l}
i \neq k \\
\mathbf{W}_{j, k} \succ 0 \quad \forall k, j \\
\operatorname{Rank}\left(\mathbf{W}_{j, k}\right) \leq 1 \quad \forall k, j .
\end{array} \quad \forall k,
\end{array}\right.
\end{aligned}
$$

Considering the semi-definite relaxation, we can rewrite problem (21) compactly as (22). The optimization problem is thus transformed into a convex approximation problem without losing optimality [[35], Chapter 4].

$$
\begin{array}{ll}
\min & \sum_{j=0}^{C} \frac{1}{\eta_{j}} \sum_{k=1}^{K} \operatorname{Tr}\left(\mathbf{W}_{j, k}\right) \\
s . t \quad & \left\{\begin{array}{l}
\left(1+\frac{1}{\gamma_{k}}\right) \sum_{j=0}^{C} \operatorname{Tr}\left(\mathbf{H}_{j, k} \mathbf{W}_{j, k}\right)-\sum_{i=1}^{K} \sum_{j=0}^{C} \operatorname{Tr}\left(\mathbf{H}_{j, k} \mathbf{W}_{j, i}\right) \geq \sigma_{k}^{2} \quad \forall k, \\
\sum_{\substack{K \\
k=1}} \operatorname{Tr}\left(\mathbf{Q}_{j, k} \mathbf{W}_{j, k}\right) \leq p_{j} \quad \forall j, \\
\mathbf{W}_{j, k} \succ 0
\end{array} \quad \forall j, k .\right.
\end{array} .
$$

\section{Proposed CMBF algorithms}

Based on the analysis above, the optimization problem (22) can be approximated using the Centralized Algorithm under the given constraints of the SINR of the target user and the maximum power of the antenna 
Table $1 \mathrm{CMBF}$

Centralized Algorithm-—CMBF

Input: Parameters: channels $h$, the number of antennas $N$, target SINR $\gamma$, transmission power constraint $p$, Matrix weight $Q$, and system subcarrier bandwidth $B$.

Output: C, Transmit-power, Users-Allocates

Step 1. Calculate the positive semidefinite channel matrix $\mathbf{H} . \mathbf{H}=h^{H} h$. Step 2. Solve equation (22) using the $\mathbf{C V X}$ optimization tool, select the value of $\mathbf{W}^{*}$.

Step 3. Obtain system throughput $\mathrm{C}$ and transmit energy consumption.

Step 4. Calculate the useful signal power and determine which base stations the user is serving. Get Users-Allocates.

transmission. The Centralized Algorithm solves the optimal beamforming. Under this optimization algorithm, the transmission power of each antenna of the BS does not exceed the maximum transmission power, which can guarantee that each user obtains the achieve rate while making the overall system power consumption the lowest. The Centralized Algorithm is described in Table 1.

In HetNets, EE can be enhanced by combining massive MIMO and small Cell technology. Considering the factor of massive MIMO, we design a cooperation scheme for different MBS antenna quantity. The cooperation scheme needs to traverse the number of different antennas and the number of cooperative SBSs. This scheme is implemented by Algorithm 1. Service user quantity in a cell is given and the SBS are symmetrically deployed around the MBS, and the EE of the number of various cooperative BSs under different MBS antenna numbers is calculated. The Algorithm 1 is described in Table 2.

In the actual scenario, the users have different achievable rate requirements. However, most existing cooperative schemes only consider the improvement of cell transmission efficiency and spectrum utilization, while neglecting the difference in resource requirements between users. To maximize the use of resources and

Table 2 EE optimization with different number of antennas

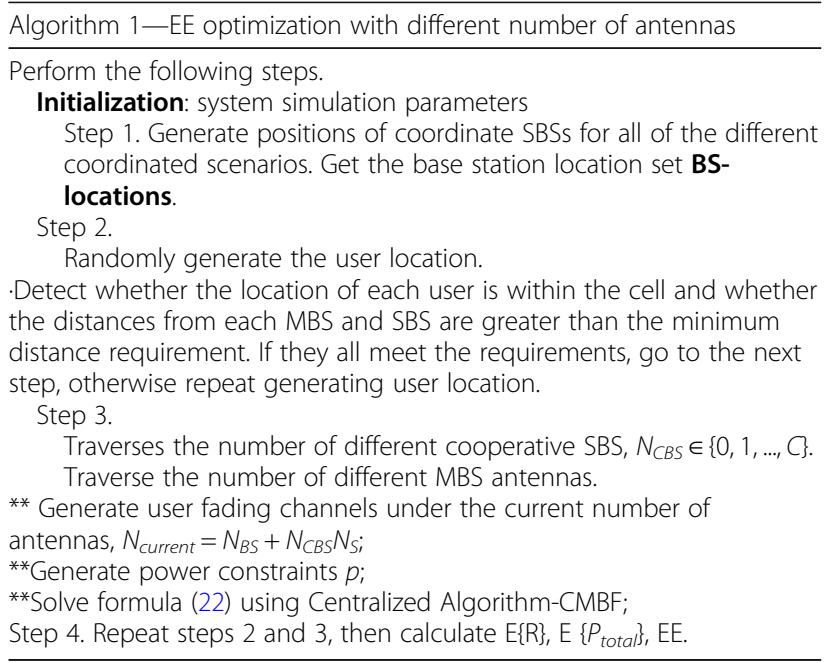

Table 3 EE optimization with different achievable rate requirements

Algorithm 2-EE optimization with different achievable rate requirements

Perform the following steps.

Initialization: system simulation parameters

Step 1. Generate positions of coordinate SBSs for all different

coordinated scenarios. Get the base station location set BS-

locations.

Step 2. implementation times is the number of Realizations Randomly generate user location.

Detect whether the location of each user is within the cell and whether the distances from each MBS and SBS are greater than the minimum distance requirement. If they all meet the requirements, go to the next step, otherwise repeat.

Traverses the number of different cooperative BS for $N_{C B S} \in\{0,1, \ldots, C$, and number of SBSs is $\mathrm{S}$.

** Calculate channels for the current number of antennas, generate user fading channels under the current number of antennas;

${ }^{*}$ Generate power constraints punder $N_{\text {currenti }}$

** Traverse the different achievable rate requirements, $R \in\{1,2,3,4\}$ with the number of antennas is $\mathrm{N}$.

***Generate user target SINR set $\gamma: \gamma=2^{R}-1$.

*** Solve formula (22) using Centralized Algorithm-CMBF;

Step 3. Calculate $E\{R\}, E\left\{P_{\text {tota }}\right\}$, EE

improve the $\mathrm{EE}$, this section contains a cooperation scheme that improves the EE based on the different achievable rate requirements of users. This solution can be generated with Algorithm 2, which makes improvements based on Algorithm 1. The process is depicted in Table 3.

After taking into account the impact of BS antenna quantity and achievable rate on the EE in the cooperative transmission of the BSs, we consider the influence of quantities of users served on EE. How many users that one BS can be served at the meantime, mainly depending on the configuration of the BS and the behavior of the users. The configuration mainly reflects the processing capability and the channel number of the BS as well as the number of carriers allocated to the cell. The user behavior mainly depends on usage habits and frequency. In this section, cooperation scheme based on the number of users is designed with a given subcarrier quantity and antenna quantity. This scheme is

Table 4 EE optimization with different number of users Algorithm 3-EE optimization with different number of users

Perform the following steps.

Initialization: system simulation parameters

Step 1. Calculate the second step in Algorithm 1 to initialize the system environment.

Step 2. Traverse the number of different cooperative $S B S, N_{C B S} \in\{0,1$, $\ldots, C\}$.

Generate power constraints.

Traverses the number of different Users equal to $10, \ldots \ldots$, max\{users\} with users $\in\{10,15 \ldots, 30\}$.

* Generate user fading channels under the current number of antennas

$N_{\text {current }}$.

${ }^{*}$ Calculate the fading channel hunder the current user number

*Solve formula (13) using Centralized Algorithm——CMBF.

Step 3. Calculate $E\{R\}, E\left\{P_{\text {total }}\right\}$, EE 


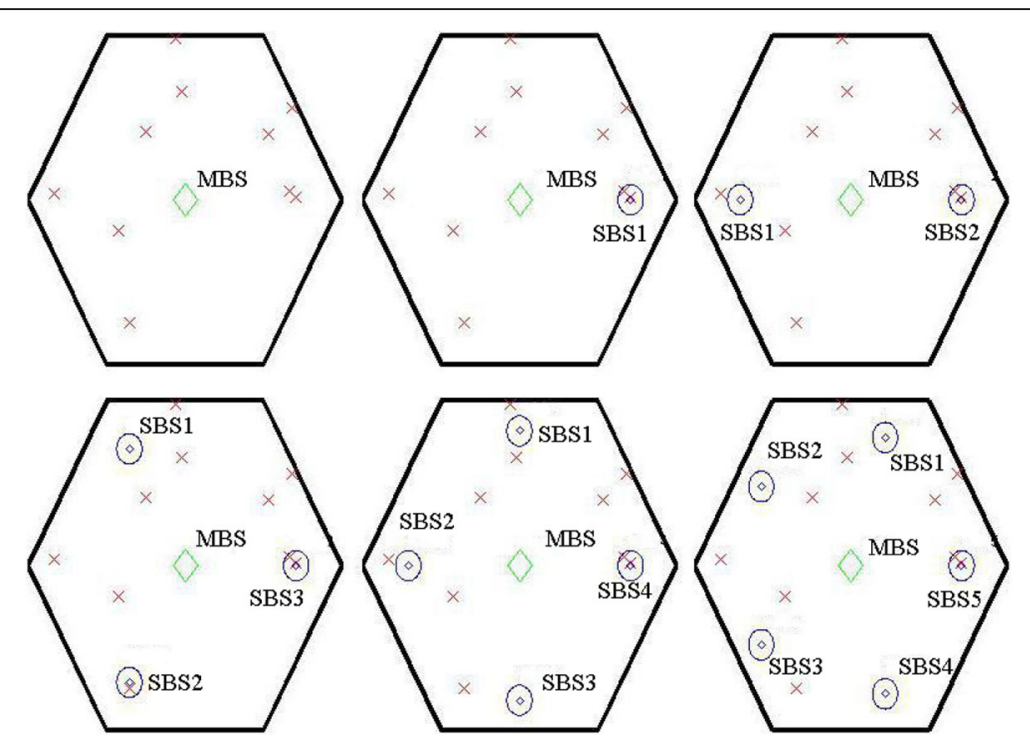

Fig. 2 Simulation model under different cooperation schemes. $\diamond$ represents macro base station. o represents small base station. $\times$ represents user

implemented with Algorithm 3. Algorithm 3 calculates the EE of the different amount of cooperative BSs under the different amount of users, given the number of BS antenna and the achievable rate. The Algorithm 3 is described in Table 4.

\section{Results and discussion}

To evaluate the performance of our designed algorithms, we provide some simulation results. We consider an LTE network for our simulation. The simulation scenario is the double-sided HetNets of regular hexagons, which coverage with numbers of SBSs. The SBSs are located $350 \mathrm{~m}$ from the center and are evenly deployed in the cell. The minimum spacing between SBSs is $40 \mathrm{~m}$, and the number of cooperating SBSs is $N_{\mathrm{CBS}} \in\{0,1, \cdots$, 10\}. There are different numbers of cooperative SBSs in the small cell, and users are randomly deployed, as shown in Fig. 2.

Table 5 Key parameters

\begin{tabular}{ll}
\hline Parameter types & Value \\
\hline Macro cell radius & $R=500 \mathrm{~m}$ \\
Small cell radius & $R=40 \mathrm{~m}$ \\
Total system bandwidth & $10 \mathrm{MHz}$ \\
Subcarrier number & 600 \\
Subcarrier bandwidth & $15 \mathrm{KHz}$ \\
Noise covariance & $-127 \mathrm{dBm}$ \\
MBS circuit power & $\rho_{0}=189 \mathrm{~mW}$ \\
SBS circuit power & $\rho_{j}=5.6 \mathrm{~mW}, \forall j \neq 0$ \\
Efficiency of MBS power amplifier & $\eta_{0}=0.388$ \\
Efficiency of SBS power amplifier & $\eta_{j}=0.052, \forall j \neq 0$ \\
\hline
\end{tabular}

The channel we assumed is a Rayleigh small-scale fading channel and the path loss model is a non-line-ofsight (NLOS) model. Table 5 shows the key system parameters for use. Most research analyzing the EE has focused on the effect of the dynamic transmission power. The static power supply power consumption, which depends on the transceiver hardware, is usually ignored. However, it needs to be considered in massive MIMO and SBSs cooperation scenarios [25]. Therefore, the EE of massive MIMO and SBSs are improved by studying the static power consumption.

This research is based on five assumptions:

- The coverage area of the MBS is hexagonal, and the SBS is randomly and uniformly deployed in the MBS coverage area.

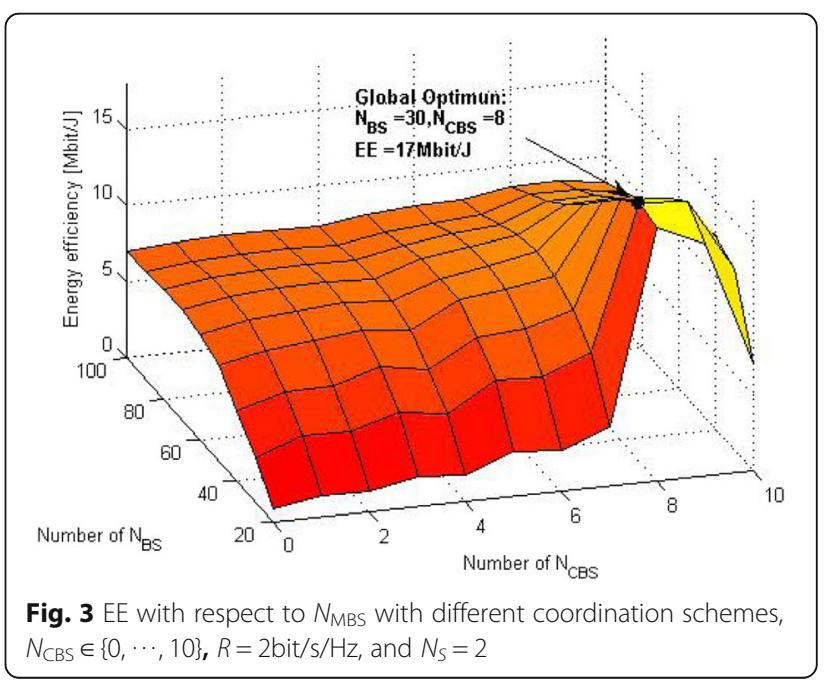




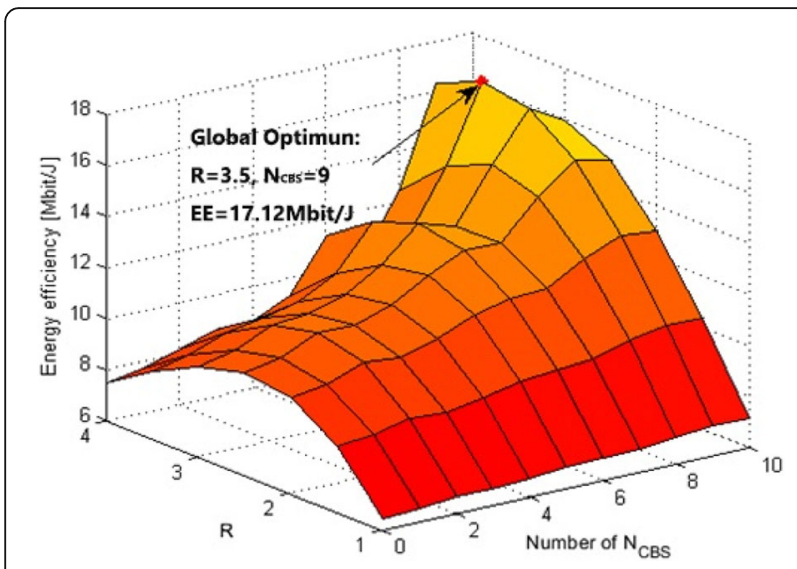

Fig. 4 EE with respect to different achievable rate constraints for $N_{S}=2, N_{C B S} \in\{0, \cdots, 10\}$, and $N_{B S}=64$

- The antennas configured by MBS and each SBS are $N_{\mathrm{BS}}$ and $N_{s}$, respectively, and they serve $k$ single antenna users.

- The transmission powers of the MBS and the SBS are different, and all SBSs have the same transmission power.

- For purpose of maximize the spectrum utilization, the MBS and the SBS also use the same frequency resources.

- The BS can get complete channel state information (CSI) and the system has an ideal backhaul.

In this simulation, the total power consumption is divided into two components: one component is dynamic consumption ratio with the transmitted signal power. Another component is circuit power consumption, which depends on the static part of the transceiver hardware. We analyze the possibility of improving the EE through the CMBF in a single cell for dual-layer HetNets with massive MIMO. Interestingly, the consideration the

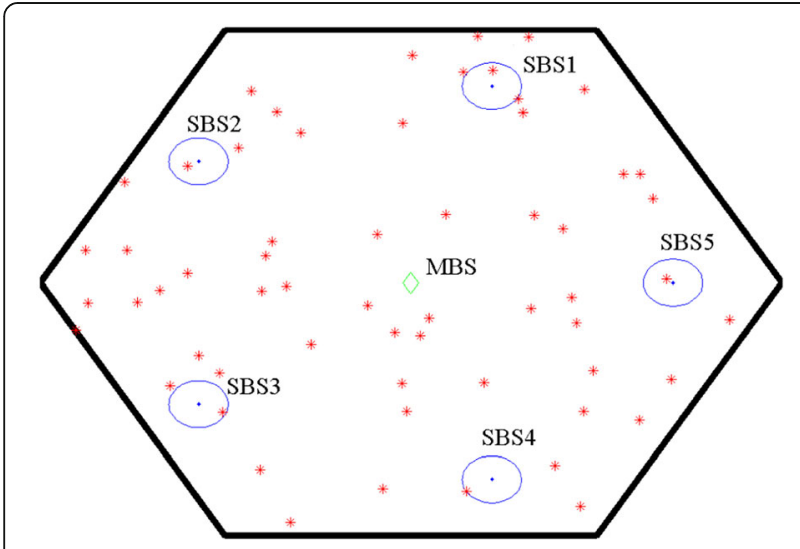

Fig. 5 Simulation model under different number of users

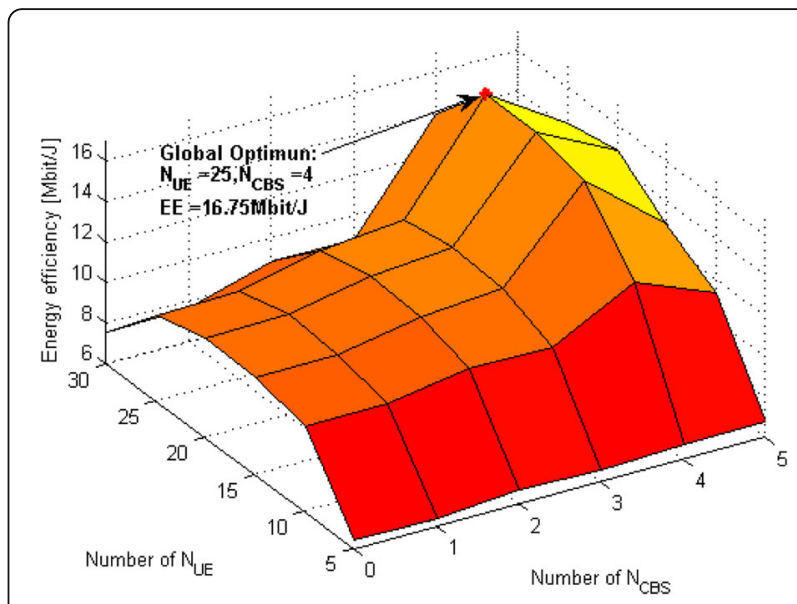

Fig. $6 \mathrm{EE}$ with different coordination schemes, $N_{U E} \in\{10,15, \cdots, 30\}$, $R=2 \mathrm{bit} / \mathrm{s} / \mathrm{Hz}$, and $N_{\mathrm{BS}}=64$

static circuit power consumption has a great influence on the EE of the massive MIMO system.

We set up 10 users and the achievable rate equal to 2 bits/s/Hz. The relationship between circuit power consumption and $N_{\mathrm{BS}}$ is shown in Fig. 3, which gives a three-dimensional perspective view of the EE. This also proves that the static circuit power consumption will reduce EE, and an appropriate number of antennas can be obtained under different cooperative SBSs and achievable rate constraints. It can be observed from Fig. 3 that massive MIMO can raise the EE and give an optimal number of $N_{\mathrm{BS}}$ needed to obtain a maximum. At the same time, SBSs and cooperation can further improve the EE. The figure illustrates the system achieves global EE-optimum when $N_{\mathrm{BS}}=30$ and $N_{\mathrm{CBS}}=8$.

For further comparison of different treatment options, Fig. 4 shows the maximum EE with achievable rate as a variable. The antenna of SBS $N_{S}=2$, and the cooperative

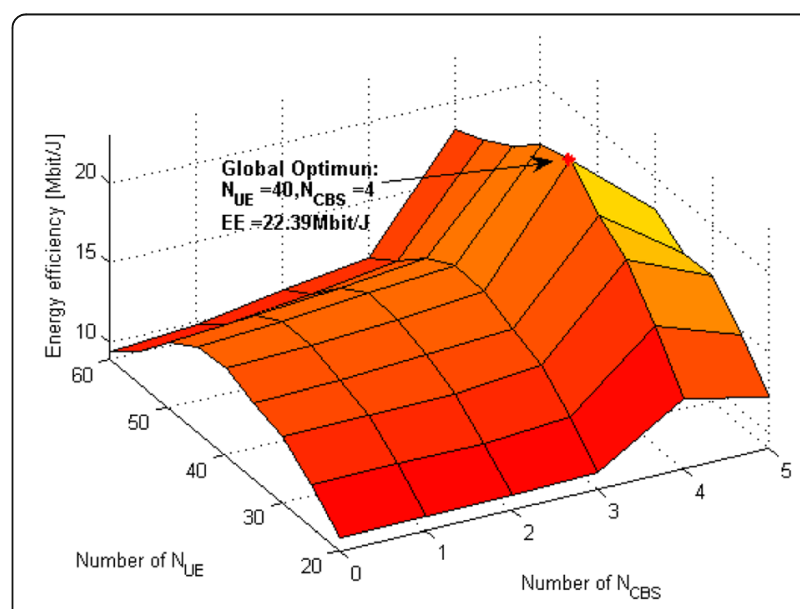

Fig. $7 \mathrm{EE}$ with different coordination schemes, $N_{U E} \in\{20,30, \cdots, 60\}$, $R=2 \mathrm{bit} / \mathrm{s} / \mathrm{Hz}$, and $\mathrm{N}_{\mathrm{BS}}=128$ 


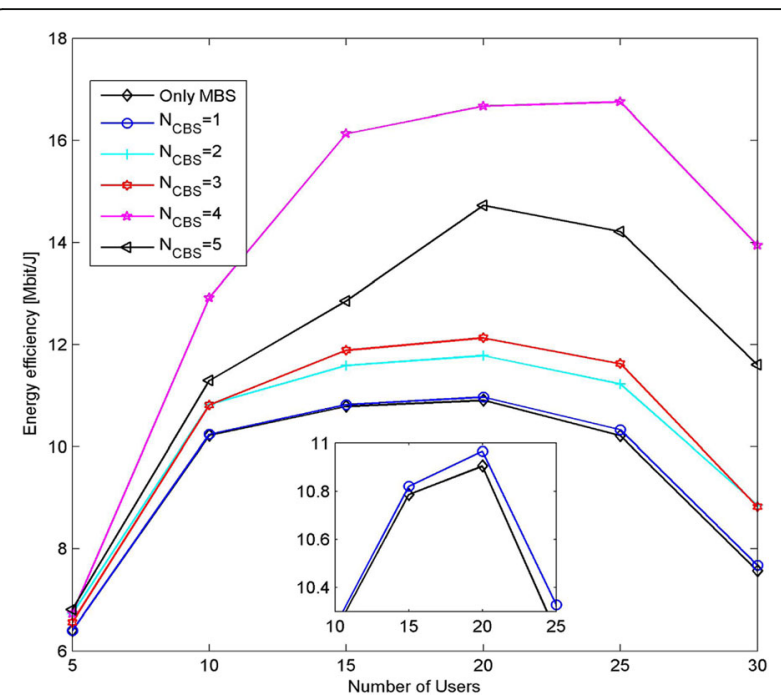

Fig. 8 EE with respect to $N_{B S}$ for different coordination schemes. $\diamond$ represents there is only macro base station that the number of small base station $N_{C B S}=0$. 0 represents the number of small base station $N_{C B S}=1$. + represents the number of small base station $N_{C B S}=2$. ${ }^{*}$ represents the number of small base station $N_{C B S}=3$. is represents the number of small base station $N_{C B S}=4$. represents the number of small base station $N_{C B S}=5$

BSs $N_{\mathrm{CBS}} \in\{0, \cdots, 10\}$, and massive MIMO antennas $N_{\mathrm{CBS}}=64$. The simulation shows that the trend of the $\mathrm{EE}$ increasing first and then decreasing with the increase of the achievable rate. It shows that the system can provide users with the best achievable rate and the highest $\mathrm{EE}$ under the condition of the given massive MIMO

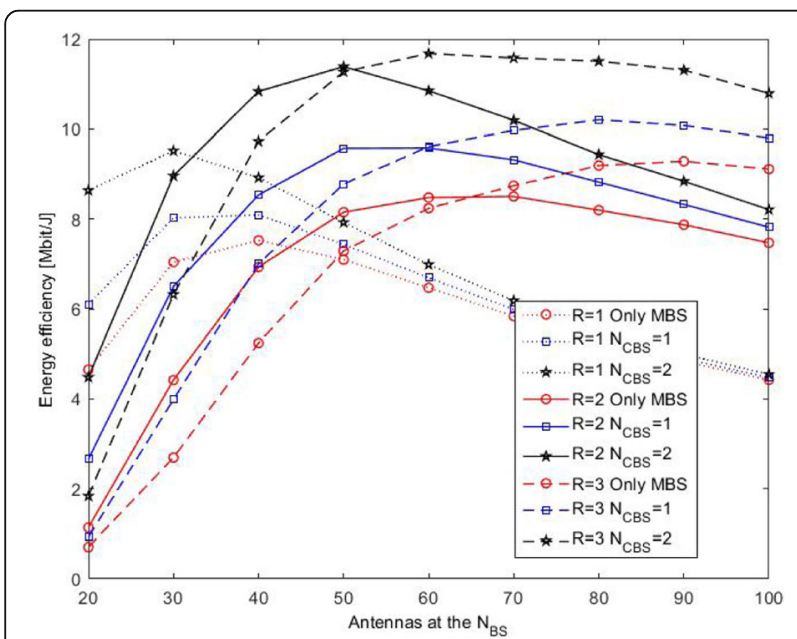

Fig. 9 EE with respect to $N_{B S}$ for different coordination schemes $N_{\text {CBS }} \in\{0,1,2\}, R \in\{1,2,3\}$, and $N_{S}=2$..... represents the achievable rate constraints of $3 \mathrm{bit} / \mathrm{s} / \mathrm{Hz}$. _ represents the achievable rate constraints of $2 \mathrm{bit} / \mathrm{s} / \mathrm{Hz}$. ..... represents the achievable rate constraints of $1 \mathrm{bit} / \mathrm{s} / \mathrm{Hz}$. o represents there is only macro base station and the number of small base station $N_{\mathrm{CBS}}=0 . \square$ represents the number of small base station $N_{\mathrm{CBS}}=1$. is represents the number of small base station $N_{C B S}=2$

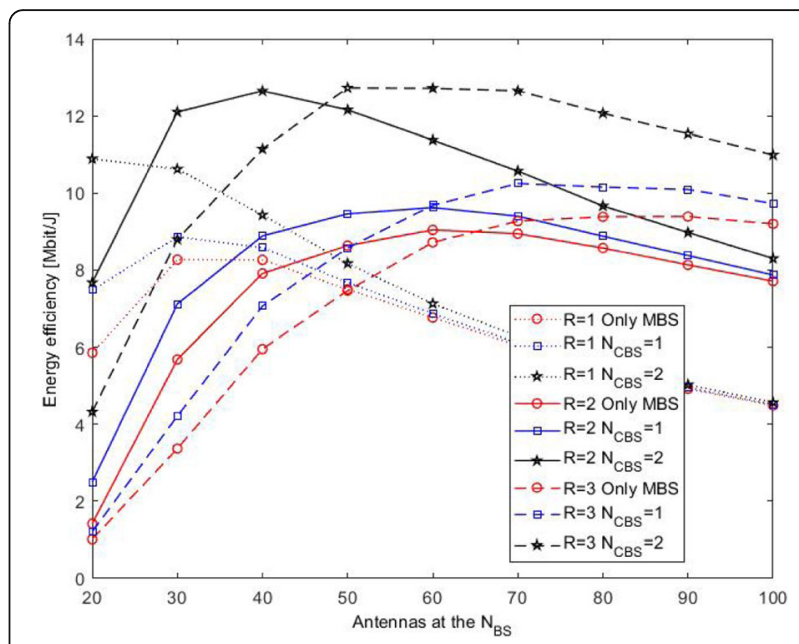

Fig. $10 \mathrm{EE}$ with respect to $N_{\mathrm{BS}}$ for different coordination schemes $N_{C B S} \in\{0,1,2\}, R \in\{1,2,3\}$, and $N_{S}=4$..... represents the achievable rate constraints of $3 \mathrm{bit} / \mathrm{s} / \mathrm{Hz}$. _ represents the achievable rate constraints of $2 \mathrm{bit} / \mathrm{s} / \mathrm{Hz}$. ..... represents the achievable rate constraints of $1 \mathrm{bit} / \mathrm{s} / \mathrm{Hz}$. o represents there is only macro base station and the number of small base station $N_{\text {CBS }}=0$. $\square$ represents the number of small base station $N_{\mathrm{CBS}}=1$. $i v$ represents the number of small base station $N_{C B S}=2$

antennas. Meanwhile, the EE can be further improved by increasing the cooperative SBSs. The EE optimum 17.12 $\mathrm{Mbit} / \mathrm{J}$ is achieved when $R=3.5$ and $N_{\mathrm{CBS}}=9$.

The simulation model under different number of users $N_{\mathrm{UE}}$ as seen in Fig. 5. Figures 6 and 7 show the impact on the EE considering the number of users and the number of cooperative BSs. Interestingly, $N_{\mathrm{BS}}=128$ processing gives a very different behavior: the EE optimum is much higher than with $N_{\mathrm{BS}}=64$ and is achieved at $N_{\mathrm{UE}}=40$ and $N_{\mathrm{CBS}}=4$. It can be seen that when quantity of BS antennas is fixed, quantity of users that the system can serve has a critical value. Specifically, there is an optimal number of service users. With this number of users, the system can guarantee the minimum achievable rate requirement of each user.

It also can be concluded that in the case of the same number of users and MBS antennas, the EE will be increased by adding quantity of deployed SBSs and cooperative SBSs. There is a critical value for the number of cooperative SBSs to make the highest EE under the circumstances of other parameters being certain (user quantity, number of antennas, achievable rate, transmission power, base station deployment mode, etc.).

Figure 7 continues to increase the number of users, while increasing the number of MBS antennas. As we can see, the properties are the same as Fig. 6, but EE is further increased. Therefore, more MBS antennas need to be equipped for user-intensive hotspots areas. Meanwhile, the $\mathrm{EE}$ varies with the number of cooperative 
SBSs. Therefore, it is necessary to select an appropriate number of cooperative SBSs according to the number of users when the number of MBS antenna is fixed for purpose of maximum EE.

Figure 8 shows that the EE of the only MBS and $N_{\text {CBS }}=1$ are very close. This is because the BS is jointly serving a certain user under the condition that the power and the achievable rate constraints are satisfied. If it is not satisfied (that is, if an unrealizable situation occurs, the CVX optimization will fail to calculate a condition that satisfies both the constraint of power and the minimum power consumption under achievable rate), and BSs will not cooperate.

There are two situations:

1. Only MBS serves users. The power of the SBS does not meet the service requirements (e.g., power, achievable rate) and does not serve the user.

2. It is only served by a single SBS. For example, in an edge area, the MBS may not serve the user (achievable rate cannot reach the user service requirement).

Therefore, the MBS may not participate in cooperation or serve the user when the constraint condition is not satisfied and the number of SBSs is 1, as in Fig. 8. Then the calculation of the power consumption for $N_{\mathrm{CBS}}=1$ includes the sum of the circuit power consumption of the MBS (transmission power consumption of MBS is equal to zero, since it does not serve a certain user) and the transmission power consumption and circuit power consumption of the SBS. The power consumption of the curve. OnlyMBS is the sum of the transmission power and the circuit power consumption of the MBS. Since the transmission power and the circuit power consumption of the SBS are relatively small, the power consumption of the curve OnlyMBS is larger than that of $N_{\mathrm{CBS}}=$ 1. Moreover, because the optimization problem of this algorithm involves guaranteeing the minimum achievable rate, when the number of users is the same, the throughput of systems using different cooperative schemes is the same. Therefore, the curve OnlyMBS and the curve $N_{\mathrm{CBS}}=1$ are relatively close, and the ordinate of the curve $N_{\mathrm{CBS}}=1$ is slightly higher.

The EE of the system determined by the different achievable rate of users and cooperative SBS quantity is shown in Figs. 9 and 10. With $N_{S} \in\{2,4\}, R \in\{1,2,3\}$, $N_{\text {CBS }} \in\{0,1,2\}$, the number of users is 10 . Results illustrate that the EE can be raised by the optimal setting of these parameters in the system design, by analyzing the different number of antennas, the achievable rate constraints, and the cooperative base station. It has also been found that the number of coordinated SBSs can be increased, and fewer MBS antennas can meet the achievable rate with the same EE. Additionally, compared to Fig. 9, the EE in Fig. 10 can be further raised by adding antenna quantity of the cooperative SBSs.

\section{Conclusion and future work}

In this paper, we studied the EE of dual-layer heterogeneous coordinated multipoint networks with massive MIMO. Research shows that the combination of massive MIMO and SBSs deployment can effectively improve the EE. Meanwhile, the EE analysis of the massive MIMO system is performed by comprehensively considering the total energy consumption of the transmission power and the circuit power. The system design is performed by considering the influence of factors such as the cooperation scheme of the SBSs, the number of antennas of the $\mathrm{BS}$, the achievable rate constraints, and the user quantity on the system EE. The analysis and simulation results illustrate that the EE can be further optimized by using $\mathrm{CMBF}$ and considering the different cooperative schemes with the antenna configurations in dual-layer HetNets with massive MIMO. The content of this paper has important guiding significance for the design of HetNets. The results in this paper encourage several future extensions such as exploiting mmWave hybrid beamforming, the investigation of deep learning models for heterogeneous massive MIMO systems.

\section{Abbreviations \\ CMBF: Coordinated multipoint beamforming; EE: Energy efficiency; MIMO: Multiple input multiple output; BS: Base station; MBS: Macro base station; SBS: Small base station; QoS: Quality of service; SCs: Small cells; SE: Spectral efficiency; CoMP: Coordinated multipoint; ICT: Information and communication technology; ICl: Inter-cell interference; CSI: Channel state information; SINR: Signal-to-interference-plus-noise ratio; PA: Power amplifiers;: SDP: Semi-definite programmingQCQPQuadratic constrained quadratic program}

\section{Acknowledgements}

The authors are grateful to the National Science Foundation of China and Natural Science Foundation of Inner Mongolia Autonomous Region of China for their support of this research.

\section{Authors' contributions}

The algorithms proposed in this paper have been conceived by Dr. Yinghui Zhang and Dr. Yang Liu. Dr. Yinghui Zhang, Dr. Yang Liu and Dr. Jing Gao designed the experiments. B.S. Biao Zhang, B.S. Yifan Mu and B.S. Kun Song performed the experiments and analyzed the results. Dr. Yinghui Zhang wrote the paper. The authors read and approved the final manuscript.

\section{Funding}

This work was supported in part by the National Natural Science Foundation of China (NSFC) under Grant 61761033, Supported by Natural Science Foundation of Inner Mongolia Autonomous Region of China under Grant 2016MS0604.

Availability of data and materials

Not applicable.

Competing interests

The authors declare that they have no competing interests. 


\section{Author details}

${ }^{1}$ College of Electronic Information Engineering, Inner Mongolia University, Hohhot, Inner Mongolia, China. ${ }^{2}$ Tianjin Key Laboratory of Wireless Mobile Communications and Power Transmission, Tianjin Normal University, Tianjin, China.

Received: 6 September 2019 Accepted: 13 March 2020

Published online: 30 March 2020

\section{References}

1. J.G. Andrews, S. Buzzi, W. Choi, S.V. Hanly, ALACK Soong, JC Zhang, What will 5 G be? IEEE J. Sel. Areas Commun. 32(6), 1065-1082 (2014)

2. J Hoydis, M Kobayashi M Debbah, Green small-cell networks. IEEE Vehicular Technol. 6(1), 37-43(2011).

3. V Jungnickel, K Manolakis, W Zirwas et al., The role of small cells, coordinated multipoint, and massive MIMO in 5G. IEEE Commun Mag. 52(5), 44-51(2014).

4. YS Soh, TQS Quek, M. Kountouris et al., Energy efficient heterogeneous cellular networks. IEEE J. Sel. Areas Commun. 6(1), 840-850(2013).

5. D Feng, C Jiang, G Lim et al., A survey of energy-efficient wireless communications. IEEE Commun. Surv. Tutor. 15(1), 167-178(2013).

6. RLG Cavalcante, S Stanczak, M Schubert et al., Toward energy-efficient 5G wireless communications technologies: tools for decoupling the scaling of networks from the growth of operating power, IEEE Signal Process. Mag. 31(6), 24-34(2014).

7. N Fatema, G Hua, Y Xiang et al., Massive MIMO linear precoding: a survey. IEEE system. Journal, 12(4), 3920-3931(2018).

8. AF Molisch, W Ratnam, S Han et al., Hybrid beamforming for massive MIMO: a survey. IEEE commun. Mag. 55(9), 134-141(2017).

9. Y Ramamoorthi, A Kumar, Resource allocation for CoMP in cellular networks with base station sleeping. IEEE Access. 6, 12620-12633(2018).

10. G Nigam, P Minero M Haenggi, Coordinated multipoint joint transmission in heterogeneous networks. IEEE Trans. Commun. 62(11), 4134-4146(2014).

11. M Avgerinou, P Bertoldi L Castellazzi, Trends in data centre energy consumption under the european code of conduct for data centre energy efficiency. Energies. 10(10), 1470(2017).

12. M Yemini, A Zappone, E Jorswieck et al., Energy efficient bidirectional massive MIMO relay beamforming. IEEE Signal Process. Lett. 24(7), 4060(2017).

13. GY Li, Z Xu, C Xiong et al., Energy-efficient wireless communications: tutorial, survey, and open issues. IEEE Wireless Commun. 18(6), 28-35(2011).

14. H Zhang, H Liu, J Cheng, VCM Leung, Downlink energy efficiency of power allocation and wireless backhaul bandwidth allocation in heterogeneous small cell networks. IEEE Trans. Commun. 66(4), 1705-1716(2018).

15. N Zhao, FR Yu, H Sun, Adaptive energy-efficient power allocation in green interference-alignment-based wireless networks. IEEE Trans. Veh. Technol. 64(9), 4268-4281(2015).

16. D.W.K. Ng, E.S. Lo, R. Schober, Energy-efficient resource allocation in OFDMA systems with large numbers of base station antennas. IEEE Trans. Wireless Commun. 11(9), 3292-3304 (2012)

17. Z Hasan, H Boostanimehr, VK Bhargava, Green cellular networks: a survey, some research issues and challenges. IEEE Commun Surv Tut. 13(1), 524540(2011).

18. Y Huang, CW Tan, BD Rao, Joint beamforming and power control in coordinated multicell: max-min duality, effective network and large system transition. IEEE Trans. Wireless Commun. 12(6), 2730-2742(2013).

19. H. Yang, G Gerac TQS Quek, Energy-efficient design of MIMO heterogeneous networks with wireless backhaul. IEEE Trans. Wireless Commun. 15(7), 4914-4927(2016)

20. A Zappone, L Sanguinetti, G Bacci et al., Energy-efficient power control: a look at $5 \mathrm{G}$ wireless technologies. IEEE Trans. Signal Process. 64(7), 16681683(2016).

21. E Björnson, M Kountouris, M Debbah, in 20st International Conference on Telecommunications (ICT). Massive MIMO and small cells: improving energy efficiency by optimal soft-cell coordination (IEEE, 2013), pp. 1-5.

22. LF Lin, WH Chung, HJ Chen, TS Lee, in 2016 IEEE International Workshop on Signal Processing Systems (SiPS). Energy efficient hybrid precoding for multiuser massive MIMO systems using low-resolution ADCs (IEEE, 2016), pp. 115120.
23. Z Chang, Z Wang, X Guo, et al., in IEEE Global Communications Conference (GLOBECOM). Energy efficient resource allocation for wireless power transfer enabled massive MIMO system (IEEE, 2016), pp. 1-7.

24. AJ Fehske, F Richter, GP Fettweis, in IEEE Globecom Workshops. Energy efficiency improvements through micro sites in cellular mobile radio networks (IEEE, 2009), pp. 1-5.

25. H Yang, T L Marzetta, in IEEE 81st Vehicular Technology Conference (VTC Spring). Energy efficient design of massive MIMO: how many antennas? (IEEE, 2015), pp. 1-5.

26. A Puglielli, A Townley, G Lacaille et al., Design of energy and cost efficient massive MIMO arrays. Proceedings of the IEEE. 104(3), 586-609(2016).

27. G Xu, A Liu, W Jiang et al., Energy-efficient beamforming for two-tier massive MIMO downlink. china commun. 12(10), 64-75(2015).

28. J Mei, L Zhao, K Zheng et al., Energy-efficient dual-layer coordinated beamforming scheme in multi-cell massive multiple-input-multiple-output systems. IET commun. 11(1), 30-38(2017).

29. G Xu, C Lin, W Ma et al., Outage constrained robust hybrid coordinated beamforming for massive MIMO enabled heterogeneous cellular networks. IEEE Access. 5, 13601-13616(2017).

30. LD Nguyen, HD Tuan, TQ Duong, et al., Downlink beamforming for energyefficient heterogeneous networks with massive MIMO and small cells. IEEE Trans. Wireless Commun. 17(5), 3386-3400(2018).

31. J Choi, On Downlink beamforming with small cells in wireless heterogeneous systems. IEEE Wireless Commun. Lett. 4(4), 425-428(2015).

32. G Auer, V Giannini, C Densset et al., How much energy is needed to run a wireless network?. IEEE Trans. Wireless Commun. 18(5), 40-49(2011).

33. $\mathrm{D} \mathrm{Ha}, \mathrm{K}$ Lee, J Kang, in IEEE 24th International Symposium on Personal Indoor and Mobile Radio Communications (PIMRC). Energy efficiency analysis with circuit power consumption in massive MIMO systems (IEEE,2013), pp. 938942.

34. ZQ Luo, WK Ma,AM So et al., Semidefinite relaxation of quadratic optimization problems. IEEE Signal Process. Mag. 27(3), 20-34(2010).

35. D.P. Palomar, Y.C. Eldar, Convex optimization in signal processing and communications (Cambridge Univ. Press, Cambridge, U.K., 2009)

\section{Publisher's Note}

Springer Nature remains neutral with regard to jurisdictional claims in published maps and institutional affiliations.

\section{Submit your manuscript to a SpringerOpen ${ }^{\circ}$ journal and benefit from:}

- Convenient online submission

- Rigorous peer review

- Open access: articles freely available online

- High visibility within the field

- Retaining the copyright to your article

Submit your next manuscript at $\boldsymbol{\nabla}$ springeropen.com 